\title{
Nonequilibrium Reduced-Density Operator for Nanoscopic Systems
}

\author{
Z. JACYNA-ONYSZKIEWICZ* \\ Quantum Physics Division, Faculty of Physics \\ A. Mickiewicz University, Umultowska 85, 61-614 Poznań, Poland
}

(Received July 22, 2002)

\begin{abstract}
Retarded forms of the nonequilibrium reduced-density operator are derived from the generalized Schrödinger variational principle of a system with both mechanical and thermal perturbations, taking into account the additional condition that the values of generalized thermodynamic coordinates are fixed at infinitely remote past. This reduced density operator may be useful in describing nonequilibrium properties of nanoscopic and mesoscopic systems, as well as ultrathin films. With the help of this reduced-density operator the generalized Green-Kubo formulae are obtained.
\end{abstract}

PACS numbers: 05.30.-d, 05.70.Ln

\section{Introduction}

Ultrathin films, mesoscopic and nanoscopic systems are frequently subjects of interests in research as well as micro- and nanoelectronics and often the need arises to take into regard their interactions with environment. For such systems the postulates of quantum statistical mechanics are not exactly fulfilled as this theory assumes that the effect of environment can only be a mixing of states in the system studied and that it has no influence on the spectrum of eigenstates of its Hamiltonian. The above facts instigate the need of formulation of a quantum theory of nanoscopic systems, mesosopic systems, and thin films more general than the quantum statistical mechanics.

Usually, while investigating theoretically macroscopic bulk systems we do not have to take into account their interaction with environment regarding the short-range character of intermolecular interactions. Any effective interaction

*e-mail: zbigonys@main.amu.edu.pl 
among the molecules of the system and its environment could only occur through the atoms on the system surface or near it. The number of the interacting atoms usually is an insignificant part of all atoms of the system. However, considering thin films and alike systems, the number of atoms interacting with environment is frequently of the same order of magnitude as the total number of atoms in the system. Moreover, thin films are deposited on bulk substrates whose structure affects that of the films, so the interaction between the substrate and the film has to be taken into regard. A common procedure to realize it is to introduce phenomenological surface parameters. The values of these parameters significantly influence physical properties of nanoscopic and mesoscopic systems or thin films which is for example evidenced in their spectra of collective excitations.

In this paper we propose a general method which takes into account the interaction between the nanoscopic system or thin film and the substrate on a microscopic level. In this method the interactions are included already in the construction of the nonequilibrium reduced-density operator which makes it possible to apply the mean field type approximation. The method proposed is a generalization of the ideas presented earlier [1-3].

\section{Fundamental assumptions and nonequilibrium reduced-density operator}

It is known that the only many-body system which can be treated as a fully isolated system is the Universe itself. All other many-body systems must be considered as non-isolated and thus interacting with environment, which means that they cannot be exactly described within the quantum theory. The need to describe the Universe as a closed system within the quantum theory gave rise to a new field of theoretical physics called quantum cosmology. In quantum cosmology the Universe is understood as the greatest physical system describable in terms of the quantum theory. However, quantum cosmology has generated some difficult interpretation problems in the quantum theory. New interpretations have appeared, satisfactory from the point of view of the quantum cosmology. Quantum cosmology assumes that the whole Universe can be described in terms of a state vector. We have taken this assumption which means we assume that the notion of the state vector of the Universe is meaningful as it is implied by the recently proposed interpretation of the quantum theory [4-6].

We assume that at the time $t=-\infty$ (in a far past for a given system) the state vector of the Universe satisfies the stationary Schrödinger equation:

$$
H|\Psi(t=-\infty)\rangle=E|\Psi(t=-\infty)\rangle,
$$

where $H$ is the Hamiltonian of the Universe. It can be easily shown that this equation is equivalent to the following variational principle:

$$
\delta \operatorname{Tr}[(H-E) d(t=-\infty)]=0,
$$


where

$$
d(t=-\infty)=|\Psi(t=-\infty)\rangle\langle\Psi(t=-\infty)|
$$

is the density operator of the Universe.

Next we divide the Universe into three subsystems: the nanoscopic system studied 1, its closest macroscopic environment 2, and the remaining part of the Universe 3 , so the Hamiltonian $H$ can be written as

$$
H=\sum_{j=1}^{3} H_{j}+\sum_{j \neq j^{\prime}} H_{j j^{\prime}},
$$

where $H_{j}$ is the Hamiltonian of the systems $j=1,2,3$, and the terms $H_{j j^{\prime}}$ describe the interactions among the systems.

The expectation value $\left\langle a_{j}\right\rangle$ of a given observable $a_{j}$ of the systems $j=1,2,3$ is given by the following formula [7]:

$$
\left\langle a_{j}\right\rangle=\left\langle\Psi\left|a_{j}\right| \Psi\right\rangle=\operatorname{Tr}\left[a_{j} d\right]=\operatorname{Tr}\left[a_{j} d_{j}\right],
$$

where

$$
d_{j}=\operatorname{Tr}_{j^{\prime}+j^{\prime \prime}}[d], \quad j^{\prime}, j^{\prime \prime} \neq j
$$

is the reduced-density operator of the system $j$, while $\operatorname{Tr}_{j^{\prime}+j^{\prime \prime}}$ [] is the partial trace over the states of the system $j^{\prime}+j^{\prime \prime}$ (of the remaining part of the Universe).

The dimensionless von Neumann entropy of the system $j$, describing the degree of mixing of its states, is defined by the following equation [7]:

$$
s_{j}=-\operatorname{Tr}_{j}\left[d_{j} \ln d_{j}\right] .
$$

We assume that for $t=-\infty$, the degree of state mixing is fixed for all three systems, so

$$
s_{j}(t=-\infty)=-\operatorname{Tr}_{j}\left[d_{j}(t=-\infty) \ln d_{j}(t=-\infty)\right]=\operatorname{constant}\left[d_{j}\right]>0
$$

for $j=1,2,3$.

In a nanoscopic system 1 nonequilibrium processes can occur as a result of the system's response to the switching-on of an external time-dependent classical field (mechanical perturbations) or as a result of the internal inhomogeneities in the nanoscopic system (thermal perturbation). Till now, in most cases these two types of sources of nonequilibrium processes have been investigated separately. However, the division of perturbations into mechanical and thermal is, in general, justified only in the first approximation. In higher approximations, mechanical perturbations create inhomogeneities in the distributions of mass, energy, and momentum and, consequently, lead to the appearance of thermal perturbations $[1,8,9]$.

The paper presented is aimed at a construction of a general formalism which can describe both types of mechanical and thermal perturbation processes in nanoscopic system 1. 
We shall consider the response of the nanoscopic system 1 with the Hamiltonian $H_{1}$ independent of time $t$, to the adiabatic switching-on of a time-dependent external small perturbation $V_{1 \delta}(t)$. The total Hamiltonian of the system 1, including the external perturbation, is

$$
H_{1}(t)=H_{1}+V_{1 \delta}(t)
$$

where $V_{1 \delta}(t)$ is the operator of interaction of a given nanoscopic system 1 with the external classical field. We assume that at $t=-\infty$ an external perturbation occurred

$$
\begin{aligned}
& V_{1 \delta}(t=-\infty)=0, \\
& V_{1 \delta}(t)=\nu_{1}(t) \exp (\delta t), \quad \delta \rightarrow+0 .
\end{aligned}
$$

To develop a mechanics of nonquilibrium processes such that the thermal perturbations are also included, it is necessary to construct a reduced-density operator $d_{1}(t)$ representing the conditions in which the nanoscopic systems are found. This turns out to be possible, if we are interested in the behavior of the system 1 in time intervals which are not too short, when the details of the initial state of the system 1 are unimportant and the number of parameters necessary for the description of the nonequilibrium state of the nanoscopic system 1 is reduced. Let the nonequilibrium state of a system 1 in the time $t+t^{\prime}$ be defined by a set of the expectation values [8]

$$
\left\langle O_{1 n}\right\rangle\left(t+t^{\prime}\right)=\operatorname{Tr}_{1}\left[O_{1 n} d_{1}\left(t+t^{\prime}\right)\right]=\operatorname{constant}\left[d_{1}\right]
$$

of certain operators $O_{1 n}$, where $n$ is an index which can take continuous or discrete values. As a consequence, we come to the condition

$$
\delta\left\langle O_{1 n}\right\rangle\left(t+t^{\prime}\right)=\operatorname{Tr}_{1}\left[O_{1 n} \delta d_{1}\left(t+t^{\prime}\right)\right]=0,
$$

where $\delta d_{1}$ is the first variation of the reduced-density operator. The reasons for introducing the time $t+t^{\prime}$ is the same as in Refs. $[1,8]$.

To describe the hydrostatic stage of nonequilibrium processes in the nanoscopic system 1 we must choose as $O_{1 n}$ operators those of the energy density, momentum, and particle number, or their Fourier components. To describe the kinetic stage of nonequilibrium processes we can choose as $O_{1 n}$ the operator of occupation numbers of the one-particle states.

So, we are looking for the extremum of the functional (2) in time $t=-\infty$ under the supplementary conditions (8) and (12). In fact, the conditional extremum in the functional (2) corresponds to the unconditional extremum of the functional

$$
\begin{gathered}
\delta\left\{\operatorname{Tr}[(H-E) d(t=-\infty)]+\sum_{n} A_{n}\left(t+t^{\prime}\right)\left\langle O_{1 n}\right\rangle\left(t+t^{\prime}\right)\right. \\
\left.+\sum_{j=1}^{3} B_{j} s_{j}(t=-\infty)\right\}=0,
\end{gathered}
$$


where $A_{n}\left(t+t^{\prime}\right)$ and $B_{j}$ are indeterminate Lagrange multipliers. Now we also assume that $t^{\prime} \rightarrow-\infty$. This means that condition (12) has introduced memory effects [8] and has a dynamic character and includes information about the evolution of the nanoscopic system 1. It is convenient to rewrite this formula applying the Abel theorem $[10,11]$. As follows from this theorem (for any function $\left.f\left(t+t^{\prime}\right)\right)$

$$
\lim _{t^{\prime} \rightarrow-\infty} f\left(t+t^{\prime}\right)=\lim _{\varepsilon \rightarrow+0} \varepsilon \int_{-\infty}^{0} \exp \left(\varepsilon t^{\prime}\right) f\left(t+t^{\prime}\right) \mathrm{d} t^{\prime}
$$

(for a given $t$ and if this limit exists).

If we take into account (15), the functional (14) can be rewritten in the following form:

$$
\begin{gathered}
\delta\left\{\operatorname{Tr}[(H-E) d(t=-\infty)]+\sum_{n} \varepsilon \int_{-\infty}^{0} \exp \left(\varepsilon t^{\prime}\right) A_{n}\left(t+t^{\prime}\right)\left\langle O_{1 n}\right\rangle\left(t+t^{\prime}\right) \mathrm{d} t^{\prime}\right. \\
\left.\quad+\sum_{j=1}^{3} B_{j} s_{j}(t=-\infty)\right\}=0, \quad \varepsilon \rightarrow+0 .
\end{gathered}
$$

In view of the above, taking into regard the definition of the reduced-density operator (6) and assuming that systems 2 and 3 are macroscopic and all the time remain in good approximation in the state of a fixed state mixing degree (equilibrium state [3]) as systems practically uncorrelated with environment, we arrive at

$$
\begin{aligned}
& \operatorname{Tr}_{1}\left[\left(H_{1}+H_{1}^{\prime}+H_{1}^{\prime \prime}-E_{1}\right) \delta d_{1}(t=-\infty)\right] \\
& +\sum_{n} \varepsilon \int_{-\infty}^{0} \exp \left(\varepsilon t^{\prime}\right) A_{n}\left(t+t^{\prime}\right) \operatorname{Tr}_{1}\left[O_{1 n} \delta d_{1}\left(t+t^{\prime}\right)\right] \mathrm{d} t^{\prime} \\
& -B_{1} \delta \operatorname{Tr}_{1}\left[d_{1}(t=-\infty) \ln d_{1}(t=-\infty)\right]+\operatorname{Tr}_{2}\left[\left(H_{2}-E_{2}\right) \delta d_{2}(t=-\infty)\right] \\
& -B_{2} \delta \operatorname{Tr}_{2}\left[d_{2}(t=-\infty) \ln d_{2}(t=-\infty)\right]+\operatorname{Tr}_{3}\left[\left(H_{3}-E_{3}\right) \delta d_{3}(t=-\infty)\right] \\
& -B_{3} \delta \operatorname{Tr}_{3}\left[d_{3}(t=-\infty) \ln d_{3}(t=-\infty)\right]=0
\end{aligned}
$$

where

$$
\begin{aligned}
& E=E_{1}+E_{2}+E_{3}, \\
& H_{1}^{\prime}=\operatorname{Tr}_{2}\left[H_{12} d_{2}(t=-\infty)\right], \\
& H_{1}^{\prime \prime}=\operatorname{Tr}_{2}\left[H_{12} K_{12} d_{2}(t=-\infty)\right] .
\end{aligned}
$$

The quantity $K_{12}$ is a reduced correlation superoperator

$$
K_{12}=\operatorname{Tr}_{3}\left[K d_{3}\right]
$$


where the correlation superoperator $K$ is defined by the following expression:

$$
d=K d_{1} \otimes d_{2} \otimes d_{3}
$$

As shown in $[2,3]$, an exact although formal expression for $K_{12}$ can be found.

We note that this functional depends on the reduced-density operator $d_{1}$ in different moments of time. Consequently, we must transform the reduced-density operator $d_{1}\left(t+t^{\prime}\right)$ to $d_{1}(t=-\infty)$, according to the following unitary transformations:

$$
d_{1}(t=-\infty)=U_{1}^{+}\left(t+t^{\prime}, t^{\prime \prime}\right) d_{1}\left(t+t^{\prime}\right) U_{1}\left(t+t^{\prime}, t^{\prime \prime}\right), \quad t^{\prime \prime} \rightarrow-\infty
$$

for decreasing time, where

$$
U_{1}\left(t+t^{\prime}, t^{\prime \prime}\right) \cong \exp \left[-\mathrm{i}\left(H_{1}+H_{1}^{\prime}\right)\left(t+t^{\prime}-t^{\prime \prime}\right) / \hbar\right] .
$$

In expression (24) the effect of a correlation between systems 1 and 2 on the evolution of the nanoscopic system 1 has been neglected, however, the interactions between the two systems 1 and 2 in the mean field type approximation has been taken into account. It is described by $H_{1}^{\prime}$, defined by (19). Under this assumption the following relation holds:

$$
\left|\left\langle H_{1}^{\prime}\right\rangle\right| \gg\left|\left\langle H_{1}^{\prime \prime}\right\rangle\right|
$$

and in Eq. (17) the term $H_{1}^{\prime \prime}$ can be omitted.

Using (23-25) and calculating the first variations, we obtain

$$
\begin{aligned}
& d_{1 \varepsilon}(t=-\infty)=\exp \left[\Phi-\sum_{n}^{\prime} \varepsilon \int_{-\infty}^{0} \exp \left(\varepsilon t^{\prime}\right) P_{1 n}\left(t+t^{\prime}\right) U_{1}^{+}\left(t+t^{\prime}, t^{\prime \prime}\right)\right. \\
& \left.\quad \times O_{1 n} U_{1}\left(t+t^{\prime}, t^{\prime \prime}\right) \mathrm{d} t^{\prime}\right], \quad t^{\prime \prime} \rightarrow-\infty, \varepsilon \rightarrow+0 \\
& d_{2}(t=-\infty)=\exp \left[\beta_{2}\left(F_{2}-H_{2}\right)\right] \\
& d_{3}(t=-\infty)=\exp \left[\beta_{3}\left(F_{3}-H_{3}\right)\right]
\end{aligned}
$$

where

$$
\begin{aligned}
& \Phi=1-E_{1} B_{1}^{-1}, \quad P_{11}=B_{1}^{-1} \quad \text { and } \quad O_{11}=H_{1}+H_{1}^{\prime}, \\
& P_{1 n}=A_{n} B_{1}^{-1}, \quad \beta_{2} F_{2}=1-E_{2} B_{2}^{-1}, \quad \beta_{2}=B_{2}^{-1}, \\
& \beta_{3} F_{3}=1-E_{3} B_{3}^{-1}, \quad \beta_{3}=B_{3}^{-1}
\end{aligned}
$$

and $\sum^{\prime}$ stands for a summation also over $n=1$.

Equations (27) and (28) follow from the assumption that the macroscopic systems 2 and 3 in good approximation can be considered as being in the equilibrium state. The form of the reduced-density operators (27) and (28) is the same as that of the statistical operators for a canonical Gibbs distribution $[3,8]$. 
Additionally we assume that the following relations hold at any time $t$ :

$$
d_{2}(t)=d_{2}(t=-\infty)
$$

and

$$
d_{3}(t)=d_{3}(t=-\infty)
$$

As implied by the assumptions (9-11), we must transform the reduced-density operator $d_{1}(t=-\infty)$ to $d_{1}(t)$, according to the following unitary transformation for increasing time:

$$
\begin{array}{r}
d_{1 t^{\prime \prime} \delta}(t)=U_{1}\left(t^{\prime}, t^{\prime \prime}\right) W_{1 \delta}\left(t, t^{\prime \prime}\right) d_{1 \varepsilon}(t=-\infty) W_{1 \delta}^{+}\left(t, t^{\prime \prime}\right) U_{1}^{+}\left(t, t^{\prime \prime}\right) \\
t^{\prime \prime} \rightarrow-\infty, \quad \varepsilon \rightarrow+0
\end{array}
$$

where

$$
W_{1 \delta}\left(t, t^{\prime \prime}\right)=P \exp \left[\frac{1}{\mathrm{i} \hbar} \int_{t^{\prime \prime}}^{t} \widetilde{V}_{1 \delta}\left(\tau, t^{\prime \prime}\right) \mathrm{d} \tau\right], \quad \delta \rightarrow+0
$$

and

$$
\widetilde{V}_{1 \delta}\left(\tau, t^{\prime \prime}\right)=U_{1}^{+}\left(\tau, t^{\prime \prime}\right) V_{1 \delta}(\tau) U_{1}\left(\tau, t^{\prime \prime}\right)
$$

is the perturbation energy operator (11) in the Dirac picture as well as $P$ is Dyson time-ordering operator. The reduced-density operator (32) may be useful in describing nonequilibrium properties of nanoscopic systems, mesosopic systems, and ultrathin films.

For $f(a)$ - an arbitrary analytical function of the operator $a$ - the relation

$$
U f(a) U^{+}=f\left(U a U^{+}\right)
$$

holds, where $U$ is an arbitrary unitary operator. Using (35), from (32) we obtain

$$
\begin{gathered}
d_{1 t^{\prime \prime} \varepsilon \delta}(t)=\exp \left[\Phi(t)-\sum_{n}^{\prime} \varepsilon \int_{-\infty}^{0} \exp \left(\varepsilon t^{\prime}\right) P_{1 n}\left(t+t^{\prime}\right) X_{1 \delta}\left(t, t^{\prime}, t^{\prime \prime}\right)\right. \\
\left.\times O_{1 n} X_{1 \delta}^{+}\left(t, t^{\prime}, t^{\prime \prime}\right) \mathrm{d} t^{\prime}\right], \quad t^{\prime \prime} \rightarrow-\infty, \varepsilon \rightarrow+0, \delta \rightarrow+0,
\end{gathered}
$$

where

$$
X_{1 \delta}\left(t, t^{\prime}, t^{\prime \prime}\right)=U_{1}\left(t, t^{\prime \prime}\right) W_{1 \delta}\left(t, t^{\prime \prime}\right) U_{1}^{+}\left(t+t^{\prime}, t^{\prime \prime}\right)
$$

The reduced-density operator (36) can be written in a more compact form by using the notation

$$
O_{1 n=0}=1
$$

and

$$
\Phi(t)=\varepsilon \int_{-\infty}^{0} \exp \left(\varepsilon t^{\prime}\right) P_{1 n=0}\left(t+t^{\prime}\right) \mathrm{d} t^{\prime}
$$


Hence

$$
d_{1 t^{\prime \prime} \varepsilon \delta}(t)=\exp \left[-\bar{S}_{1 t^{\prime \prime} \varepsilon \delta}(t)\right], \quad t^{\prime \prime} \rightarrow-\infty, \varepsilon \rightarrow+0, \delta \rightarrow+0,
$$

where

$$
\begin{aligned}
& \bar{S}_{1 t^{\prime \prime} \varepsilon \delta}(t)=\varepsilon \int_{-\infty}^{0} \exp \left(\varepsilon t^{\prime}\right) S_{1 t^{\prime \prime} \delta}\left(t, t^{\prime}\right) \mathrm{d} t^{\prime} \\
& S_{1 t^{\prime \prime} \delta}\left(t, t^{\prime}\right)=\sum_{n}^{\prime \prime} P_{1 n}\left(t, t^{\prime}\right) X_{1 \delta}\left(t, t^{\prime}, t^{\prime \prime}\right) O_{1 n} X_{1 \delta}^{+}\left(t, t^{\prime}, t^{\prime \prime}\right)
\end{aligned}
$$

and $\sum^{\prime \prime}$ stands for a summation also over $n=0$.

The functional $\Phi(t)$ calculated from the condition of normalization [7]

$$
\operatorname{Tr}[d]=\operatorname{Tr}_{1}\left[d_{1}\right]=1
$$

can be expressed as

$$
\begin{aligned}
\Phi(t) & =\ln \operatorname{Tr}_{1}\left[\operatorname { e x p } \left[-\sum_{n}^{\prime} \varepsilon \int_{-\infty}^{0} \exp \left(\varepsilon t^{\prime}\right) P_{1 n}\left(t+t^{\prime}\right) U_{1}^{+}\left(t^{\prime}, t^{\prime \prime}\right)\right.\right. \\
& \left.\left.\times O_{1 n} U_{1}\left(t^{\prime}, t^{\prime \prime}\right) \mathrm{d} t^{\prime}\right]\right], \quad t^{\prime \prime} \rightarrow-\infty, \varepsilon \rightarrow+0 .
\end{aligned}
$$

The Lagrange multipliers $P_{1 n}\left(t+t^{\prime}\right)$ are determined from the equations

$$
\left\langle O_{1 n}\right\rangle(t)=\lim _{t^{\prime \prime} \rightarrow-\infty} \lim _{\delta \rightarrow+0} \lim _{\varepsilon \rightarrow+0} \operatorname{Tr}_{1}\left[O_{1 n} d_{1 t^{\prime \prime} \delta \varepsilon}(t)\right],
$$

where $\left\langle O_{1 n}\right\rangle(t)$ are the generalized thermodynamical coordinates. In the calculation of the expectation values $\left\langle O_{1 n}\right\rangle(t)$, the parameters $\delta>0$ and $\varepsilon>0$ tend to zero after the thermodynamic limit has been reached (if this limit exists for system 1 ).

In the particular case when $H_{1}^{\prime}=0$ and $V_{1 \delta}(t)=0$, i.e. when there is no interaction between a given macroscopic system and the classical field, the reduced-density operator (32) takes the form

$$
\begin{aligned}
& d_{1 t^{\prime \prime} \varepsilon}(t)=\exp \left[-\Phi(t)-\sum_{n}^{\prime} \varepsilon \int_{-\infty}^{0} \exp \left(\varepsilon t^{\prime}\right) P_{1 n}\left(t+t^{\prime}\right) U_{1}^{+}\left(t^{\prime}, t^{\prime \prime}\right)\right. \\
& \left.\times O_{1 n} U_{1}\left(t^{\prime}, t^{\prime \prime}\right) \mathrm{d} t^{\prime}\right], \quad t^{\prime \prime} \rightarrow-\infty, \varepsilon \rightarrow+0,
\end{aligned}
$$

which coincides with the nonequilibrium statistical operator (NESO) obtained earlier by Zubarev [8, 10]. NESO (46) has been applied by many authors [8] to different problems in the theory of irreversible processes in derivation of a set of equations for energy transport momentum and a number of particles in a many-component system, and in derivation of the relaxation or kinetic equations and those of the Kramers-Fokker-Planck type.

By means of the integration by parts, the nonequilibrium reduced-density operator (40) can be conveniently written in the form 


$$
\begin{aligned}
d_{1 t^{\prime \prime} \varepsilon \delta}(t)=\exp \left[-S_{1 t^{\prime \prime} \delta}\left(t, t^{\prime}=0\right)+\dot{S}_{1 t^{\prime \prime} \varepsilon \delta}(t)\right] & \\
& t^{\prime \prime} \rightarrow-\infty, \varepsilon \rightarrow+0, \delta \rightarrow+0,
\end{aligned}
$$

where $S_{1 t^{\prime \prime} \varepsilon \delta}\left(t, t^{\prime}=0\right)$ is the entropy operator and

$$
\dot{S}_{1 t^{\prime \prime} \varepsilon \delta}(t)=\int_{-\infty}^{0} \exp \left(\varepsilon t^{\prime}\right) \frac{\partial}{\partial t^{\prime}} S_{1 t^{\prime \prime} \delta}\left(t, t^{\prime}\right) \mathrm{d} t^{\prime}
$$

is the entropy production operator $[1,8]$. It is easy to show that $\dot{S}_{1 t^{\prime \prime} \varepsilon \delta}(t)$ is positive and that this fact is connected with the choice of the retarded form of the integral (15).

If in (47) we neglect the term with the entropy production operator we observe that the reduced-density operator coincides with the generalized statistical operator of the quasi (local)-equilibrium distribution (or local-nonlinear response statistical operator)

$$
d_{1 t^{\prime \prime} \delta}(t)=\exp \left[-S_{1 t^{\prime \prime} \delta}\left(t, t^{\prime}=0\right)\right], \quad t^{\prime \prime} \rightarrow-\infty, \delta \rightarrow+0 .
$$

This operator describes the nonequilibirium processes but does not describe the irreversible transport processes. In the particular case when $V_{\delta}(t)=0$, we obtain from this operator the quasi-equilibrium reduced-density operator

$$
d_{1 \mathrm{q}}(t)=\exp \left[-\Phi(t)-\sum_{n}^{\prime} P_{1 n}(t) O_{1 n}\right],
$$

which was used in a simpler form, e.g. by Mori [12] as the initial condition for the solution of von Neumann equation.

If the operators $O_{1 n}$ are integrals of motion, then

$$
\forall_{n}\left[O_{1 n}, H_{1}+H_{1}^{\prime}\right]=0
$$

and reduced-density operator (49) becomes the reduced-density operator for nonlinear response of the nanoscopic systems 1 :

$$
\begin{aligned}
\tilde{d}_{1 t^{\prime \prime} \delta}(t)=U_{1}\left(t, t^{\prime \prime}\right) W_{1 \delta}\left(t, t^{\prime \prime}\right) d_{1 \mathrm{eq}} W_{1 \delta}^{+}\left(t, t^{\prime \prime}\right) U_{1}^{+}\left(t, t^{\prime \prime}\right), & \\
t^{\prime \prime} & \rightarrow-\infty, \delta \rightarrow+0,
\end{aligned}
$$

where

$$
d_{1 \mathrm{eq}}=\exp \left(-\Phi-\sum_{n}^{\prime} P_{1 n} O_{1 n}\right)
$$

is the general form of the equilibrium reduced-density operator [3], and

$$
P_{1 n}=\varepsilon \int_{-\infty}^{0} \exp \left(\varepsilon t^{\prime}\right) P_{1 n}\left(t^{\prime}\right) \mathrm{d} t^{\prime} .
$$




\section{Generalized Green-Kubo formulae}

The Green-Kubo theory of linear response [8] may be applied when the thermal perturbations, arising as a result of the mechanical ones can be neglected, and when the medium is passive, i.e. there is no feedback and then the generation of thermal perturbations is impossible. In fact, in almost all real cases, the system receiving energy from the external field can pass it on to its surrounding. This is especially obvious if we think of the system as a distinct part of a large system, e.g. in the magnetic resonance phenomena. Even if we assume that at the initial moment the system was in equilibrium with a thermostat, this equilibrium is disturbed as a result of mechanical perturbations and consequently, thermal perturbations, which cannot be described by an external field, arise. For this reason we shall now consider the possibility of generalization of the Green-Kubo formulae to the case of the thermal perturbations.

If the perturbation $V_{1 \delta}(t)$ is small, $W_{1 \delta}\left(t, t^{\prime \prime}\right)$ can be written, in the first approximation, in the form

$$
W_{1 \delta}\left(t, t^{\prime \prime}\right)=1+\frac{1}{\mathrm{i} \hbar} \int_{t^{\prime \prime}}^{t} \widetilde{V}_{1 \delta}\left(\tau, t^{\prime \prime}\right) \mathrm{d} \tau+O\left(\widetilde{V}_{1 \delta}^{2}\right)
$$

Substituting (32) and (55) into the formula for the expectation value of any physical quantity of system 1 represented by the operator $A_{1}$,

$$
\left\langle A_{1}\right\rangle(t)=\lim _{t^{\prime \prime} \rightarrow-\infty} \lim _{\varepsilon \rightarrow+0} \lim _{\delta \rightarrow+0} \operatorname{Tr}\left[A_{1} d_{1 t^{\prime \prime} \varepsilon \delta}(t)\right],
$$

we obtain the generalized Green-Kubo formulae $[1,8]$ in the following form:

$$
\begin{aligned}
& \left\langle A_{1}\right\rangle(t)=\lim _{t^{\prime \prime} \rightarrow-\infty} \lim _{\varepsilon \rightarrow+0} \operatorname{Tr}\left[A_{1} d_{1 t^{\prime \prime} \varepsilon}(t)\right] \\
& \quad+\lim _{t^{\prime \prime} \rightarrow-\infty} \lim _{\varepsilon \rightarrow+0} \lim _{\delta \rightarrow+0} \int_{-\infty}^{+\infty}\left\langle\left\langle A_{1} \mid V_{1 \delta}\left(\tau, t^{\prime \prime}\right)\right\rangle\right\rangle_{\varepsilon} \mathrm{d} \tau,
\end{aligned}
$$

where

$$
\left\langle\left\langle A_{1} \mid V_{1 \delta}\left(\tau, t^{\prime \prime}\right)\right\rangle\right\rangle_{\varepsilon}=\frac{1}{\mathrm{i} \hbar} \Theta(t-\tau) \operatorname{Tr}_{1}\left\{\left[A_{1}, V_{1 \delta}\left(\tau, t^{\prime \prime}\right)\right]_{-} d_{1 t^{\prime \prime} \varepsilon}(t)\right\}
$$

is the nonequilibrium two-time retarded Green function and $d_{1 t^{\prime \prime} \varepsilon}(t)$ is the nonequilibrium operator given by formula (32) in the case of $V_{1 \delta}(t)=0$.

Formula (57) describes the linear retarded response of a nonequilibrium nanoscopic, mesoscopic or macroscopic system to the adiabatic switching-on of the classical time-dependent field. With the help of this expression we can describe the nonequilibrium processes which occur as a result of both mechanical and thermal perturbations at the same time, e.g. to describe the nonequilibrium irreversible transport processes or relaxation process in nanoscopic, mesoscopic or macroscopic systems during the adiabatic switching-on of an external time-dependent field. 


\section{Conclusion}

The most important point of our paper is the determination of the retarded forms of the nonequilibrium reduced-density operator (40). This operator has been derived from the generalized Schrödinger variational principle and describes a nanoscopic system with both mechanical and thermal perturbations. So, the reduced-density operator (40) may be useful in describing nonequilibrium properties of nanoscopic systems, mesosopic systems, and ultrathin films. With the help of this reduced density operator, the generalized Green-Kubo formulae (52) are obtained. In the method proposed, the interaction between the nanoscopic system and the substrate is included already in the construction of the reduced-density operator (40) which makes it possible to apply the mean field type approximation.

This work was partially supported by the State Committee for Scientific Research under grant No. 5 P03B 09120.

\section{References}

[1] Z. Onyszkiewicz, Physica A 143, 287 (1987).

[2] Z. Onyszkiewicz, Phys. Lett. A 163, 450 (1992).

[3] Z. Jacyna-Onyszkiewicz, Physica A 305, 497 (2002).

[4] Z. Onyszkiewicz, in: New Developments of Fundamental Problem in Quantum Physics, Eds. M. Ferrero, A. van der Merve, Kluwer Academic Publ., Amsterdam 1997 , p. 285.

[5] Z. Jacyna-Onyszkiewicz, Physics Essays 10, 417 (1997); 12, 397 (1999).

[6] Z. Jacyna-Onyszkiewicz, in: Proc. 5th Wigner Symposium, Vienna, August 1997, Eds. P. Kasperkovitz, D. Grau, World Scientific, Singapore 1998, p. 537.

[7] K. Blum, Density Matrix Theory and Applications, Plenum, New York 1981.

[8] D.N. Zubarev, Nonequilibrium Statistical Thermodynamics, Consultant Bureau, New York 1974.

[9] D.N. Zubarev, V. Morozov, G. Repke, Statistical Mechanics of Nonequilibrium Processes, Akademic Verlag, Berlin 1996.

[10] V.P. Kalasknikov, D.N. Zubarev, Physica 59, 314 (1972).

[11] G. Doetsch, Introduction to the Theory and Application of the Laplace Transformation, Springer, Berlin 1974.

[12] H. Mori, Phys. Rev. 112, 1829 (1958); 115, 298 (1959). 